\title{
A characterization of automorphism groups of simple $K_{3}$-groups
}

\author{
Dapeng Yu(*), Jinbao Li(**), Guiyun Chen(***), Yanheng Chen $(* * *)$
}

ABSTRACT - In this paper, a new characterization of automorphism groups of simple $K_{3}$-groups is presented.

Mathematics Subject Classification (2010). 20D05, $20 \mathrm{D} 45$.

KEYwords. Simple $K_{3}$-groups; conjugacy classes sizes; characterization.

(*) Indirizzo dell'A.: School of Mathematics and Statistics, Southwest University, Chongqing 400715, China; also affiliated with Department of Mathematics, Chongqing University of Arts and Sciences, Chongqing 402160, China.

E-mail: yudapeng0@sina.com

Supported by the Scientific and Technological Research Program of Chongqing Municipal Education Commission (Grant No. KJ111207) and the Scientific Research Foundation of Chongqing University of Arts and Sciences (Grant No. Z2013SC10).

(**) Indirizzo dell'A.: Department of Mathematics, Chongqing University of Arts and Sciences, Chongqing 402160, China.

E-mail: leejinbao25@163.com

Supported by the Scientific Research Foundation of Chongqing Municipal Science and Technology Commission (Grant No. cstc2013jcyjA00034), the Scientific and Technological Research Program of Chongqing Municipal Education Commission (Grant No. KJ131204), the Scientific Research Foundation of Yongchuan Science and Technology Commission (Grant No. Ycstc, 2013nc8006).

(***) Indirizzo dell'A.: School of Mathematics and Statistics, Southwest University, Chongqing 400715, China.

E-mail: gychen1963@163.com

Corresponding author and supported by the National Natural Science Foundation of China (Grant Nos. 11271301, 11171364).

$\left(*^{*}\right)$ Indirizzo dell'A.: School of Mathematics and Statistics, Chongqing Three Gorges University, Chongqing 404100, China.

E-mail: math_yan@126.com

Supported by the Scientific Research Foundation of Chongqing Municipal Science and Technology Commission (Grant No. cstc2014jcyjA00009), the Scientific and Technological Research Program of Chongqing Municipal Education Commission (Grant No. KJ1401006) and the Fundamental Research Funds for the Central Universities. 


\section{Introduction}

All groups considered in this paper are finite.

It is known that the set $c s(G)$ of sizes of the conjugacy classes of a finite group $G$ encodes much information about the structure of $G$. Many authors have studied the connection between arithmetical properties of $c s(G)$ and structural properties of $G$. The present paper is a contribution along this line, which is related to Thompson's conjecture (see [11, Problem 12.38] and $[2,3,4,5,12,1]$ for detail).

ConJeCtuRe 1.1. Let $G$ be a group with $Z(G)=1$ and $M$ is a nonabelian simple group satisfying that $c s(G)=c s(M)$, then $G \simeq M$.

In this conjecture, $M$ is a non-abelian simple group. Hence it seems interesting to consider the following question.

Let $G$ be a group with $Z(G)=1$ and $M$ is an almost simple group satisfying that $\operatorname{cs}(G)=c s(M)$. Then, what can we say about the structure of $G$ ?

A group $G$ is almost simple if there exists a non-abelian simple group $S$ such that $S \leqslant G \leqslant A u t(S)$. In [9, 10], the almost sporadic groups, Aut $(P S L(2, q))$ and $P G L(2, p)$ for some special cases are discussed. In this paper, we investigate the almost simple $K_{3}$-groups. A group $G$ is called a $K_{3}$ group if $\pi(G)$ consists of exactly three distinct primes. Among the known simple groups, there are exactly eight simple $K_{3}$-groups (see [7, p. 12]):

$$
A_{5}, \quad A_{6}, \quad L_{2}(7), \quad L_{2}(8), \quad L_{2}(17), \quad L_{3}(3), \quad U_{3}(3), \quad U_{4}(2) .
$$

Our main result is as follows.

THEOREM 1.2. Let $G$ be a group with $Z(G)=1$ and $S$ be one of the simple $K_{3}$-groups. Set $M=\operatorname{Aut}(S)$.

(1) If $S \in\left\{A_{5}, A_{6}, L_{2}(7), L_{2}(8), L_{2}(17), L_{3}(3), U_{3}(3)\right\}$ and $c s(G)=c s(M)$, then $G \simeq M$.

(2) If $S=U_{4}(2), c s(G)=c s(M)$ and $|G|_{p}=|M|_{p}$, then $G \simeq M$, where $|G|_{p}$ denotes the order of the Sylow $p$-subgroups of $G$ for $p \in\{2,3,5\}$.

\section{Preliminaries}

Let $G$ be a group and construct its prime graph $\Gamma(G)$ as follows: the vertices are the primes dividing the order of $G$, two vertices $p$ and $q$ are 
joined by an edge if and only if $G$ contains an element of order $p q$ (see [13]). We denote the set of all connected components of the graph $\Gamma(G)$ by $T(G)=\left\{\pi_{i}(G) \mid 1 \leqslant i \leqslant t(G)\right\}$, where $t(G)$ is the number of the connected components of $\Gamma(G)$. If the order of $G$ is even, we always assume that $2 \in \pi_{1}(G)$. For $x \in G, x^{G}$ denotes the conjugacy class in $G$ containing $x$ and $C_{G}(x)$ denotes the centralizer of $x$ in $G$. A group $G$ is called a 2-Frobenius group if $G$ has a normal series $1 \subseteq H \subseteq K \subseteq G$ such that $K$ and $G / H$ are Frobenius groups with kernels $H$ and $K / H$ respectively (see, for example [9, Definition 2.1]).

The other notation and terminologies in this paper are standard and the reader is referred to ATLAS [6] and [8] if necessary.

The following lemma is well-known. It follows from [4, Lemma 1.1] or [12, Lemma 3].

LEMma 2.1. Let $G$ and $M$ be groups satisfying $Z(G)=Z(M)=1$ and $\operatorname{cs}(G)=\operatorname{cs}(M)$. Then $\pi(G)=\pi(M)$.

LEMMA 2.2 [4, Lemma 1.4]. Let $G$ be a group with $Z(G)=1$ and $M a$ group with $t(M)>1$. Suppose that $\operatorname{cs}(G)=\operatorname{cs}(M)$. Then $|G|=|M|$.

Lemma 2.3 [4, Lemma 1.5]. Let $G$ and $M$ be groups satisfying that $|G|=|M|$ and $\operatorname{cs}(G)=\operatorname{cs}(M)$. Then $t(G)=t(M)$ and $T(G)=T(M)$.

LEMma 2.4. Suppose that $G$ is a Frobenius group of even order and $H$, $K$ are the Frobenius kernel and the Frobenius complement of $G$, respectively. Then $t(G)=2, T(G)=\{\pi(H), \pi(K)\}$ and $G$ has one of the following structures:

(i) $2 \in \pi(H)$ and all Sylow subgroups of $K$ are cyclic;

(ii) $2 \in \pi(K), H$ is an abelian group, $K$ is a solvable group, the Sylow subgroups of $K$ of odd order are cyclic groups and the Sylow 2-subgroups of $K$ are cyclic or generalized quaternion groups;

(iii) $2 \in \pi(K), H$ is abelian, and there exists a subgroup $K_{0}$ of $K$ such that

$$
\left|K: K_{0}\right| \leqslant 2, K_{0}=Z \times S L(2,5),(|Z|, 2 \times 3 \times 5)=1,
$$

and the Sylow subgroups of $Z$ are cyclic.

Proof. This is Lemma 1.6 in [4]. 
LEMMA 2.5. Let $G$ be a 2-Frobenius group of even order. Then $t(G)=2$ and $G$ has a normal series $1 \subseteq H \subseteq K \subseteq G$ such that $\pi(K / H)=\pi_{2}$, $\pi(H) \cup \pi(G / K)=\pi_{1}$, the order of $G / K$ divides the order of the automorphism group of $K / H$, and both $G / K$ and $K / H$ are cyclic. Especially, $|G / K|<|K / H|$ and $G$ is solvable.

Proof. This is Lemma 1.7 in [4].

LEMMA 2.6. Let $G$ be a group with more than one prime graph component. Then $G$ is one of the following:

(i) a Frobenius or 2-Frobenius group;

(ii) $G$ has a normal series $1 \subseteq H \subseteq K \subseteq G$, where $H$ is a nilpotent $\pi_{1}$-group, $K / H$ is a non-abelian simple group and $G / K$ is $a \pi_{1}$ group such that $|G / K|$ divides the order of the outer automorphism group of $K / H$. Besides, $\pi_{i}(K / H)=\pi_{i}(G)$ for $i \geqslant 2$.

PROOF. It follows straight forward from Lemmas 1-3 in [13], Lemma 1.5 in [3] and Lemma 7 in [5].

LEMMA 2.7 [9, Corollary 5.1]. Let $G$ be a group with $Z(G)=1$ and $M=A u t\left(L_{2}(q)\right)$, where $\Gamma(M)$ is not connected. If $\operatorname{cs}(G)=c s(M)$, then $G \simeq M$.

Lemma 2.8 [12, Lemma 4]. Suppose that $G$ is a group with $Z(G)=1$ and $p$ is a prime in $\pi(G)$ such that $p^{2}$ does not divide $\left|x^{G}\right|$ for all $x$ in $G$. Then a Sylow p-subgroup of $G$ is elementary abelian.

\section{Proof of Theorem 1.2}

LEMMA 3.1. Let $G$ be a group with $Z(G)=1$ and

$$
S \in\left\{A_{5}, L_{2}(7), L_{2}(8), L_{2}(17), L_{3}(3), U_{3}(3)\right\} .
$$

Let $M=A u t(S)$ and $\operatorname{cs}(G)=\operatorname{cs}(M)$. Then $G \simeq M$.

Proof. By [6], we have that $\Gamma(M)$ is not connected if

$$
S \in\left\{A_{5}, L_{2}(7), L_{2}(8), L_{2}(17), L_{3}(3), U_{3}(3)\right\} .
$$

Therefore, by Lemma $2.7, G \simeq M$ provided that

$$
S \in\left\{L_{2}(7), L_{2}(8), L_{2}(17)\right\} .
$$

Now, it suffices to discuss the remaining three cases. 
First, we suppose that $S=A_{5}$. Then $\Gamma(M)$ is not connected. By the hypothesis and lemma 2.2 , we obtain that $|G|=|M|$. Since $\pi_{2}(M)=\{5\}$ by [6], it follows from Lemma 2.3 that $\pi_{2}(G)=\{5\}$. Since 5 does not divide 23, by Lemma 2.4, we have $G$ is not a Frobenius group. Suppose that $G$ is a 2 -Frobenius group. Then, by Lemma $2.5, G$ has a normal series $1 \subseteq H \subseteq K \subseteq G$ such that $|K / H|=5$. Let $P$ be a Sylow 3-subgroup of $H$. Then $P$ is a normal subgroup of $G$ of order 3 . Let $x \in G$ such that $|x|=5$. Then $x$ acts trivially on $P$ and so $\Gamma(G)$ is connected. This contradiction shows that $G$ is not a 2-Frobenius group either. Now, by lemma 2.6, $G$ has a normal series $1 \subseteq H \subseteq K \subseteq G$ such that $K / H$ is a non-abelian simple group. It is easy to see that $K / H$ is isomorphic to $A_{5}$. If $H \neq 1$, then $H$ is of order 2 and so $H \leqslant Z(G)$, contrary to our assumption for $G$. Hence $H=1$ and therefore $K \simeq A_{5}$. It follows that $G \simeq S_{5}$, as desired.

Next, we assume that $S=L_{3}(3)$. Since $\Gamma(M)=\Gamma(A u t(S))$ is not connected, Lemma 2.2 together with the hypothesis imply that $|G|=|M|$. By [6], we have that $\{13\}$ is a component of $\Gamma(M)$. Hence, by Lemma 2.3, $\{13\}$ is a component of $\Gamma(G)$. Similar to above discussion, we can show that $G$ is not a Frobenius group. We assert that $G$ is not a 2-Frobenius group either. If not, then, by Lemma 2.5, $G$ has a normal series $1 \subseteq H \subseteq K \subseteq G$ such that $|K / H|=13$. Let $P$ be a Sylow 2-subgroup of $H$ and $\Omega=\Omega_{1}(Z(P))$. Since $\left(13,\left|G L_{n}(2)\right|\right)=1$, where $n=2,3,4,5$, an element $x$ of $G$ of order 13 act trivially on $\Omega$. Therefore $G$ has an element of order 26 , a contradiction. Thus $G$ is not a 2-Frobenius group. By Lemma 2.6, $G$ has a chief factor $K / H$ such that $K / H$ is a simple $K_{3}$-group and $13 \in \pi(K / H)$. By [6], $K / H$ must be isomorphic to $L_{3}(3)$. Similarly as above, we obtain that $H=1$ and so $G \simeq M=A u t\left(L_{3}(3)\right)$.

Finally, we prove that $G \simeq M=A u t\left(U_{3}(3)\right)$. As above, one can show that $G$ is neither a Frobenius group nor a 2-Frobenius group. By [6], $\pi_{2}(M)=\{7\}$ and so $\pi_{2}(G)=\{7\}$ by Lemma 2.3. It follows from Lemma 2.6 that $G$ has a chief factor $K / H$ such that $K / H$ is a simple $K_{3}$-group and $7 \in \pi(K / H)$. Then $K / H$ is isomorphic to $L_{2}(7), L_{2}(8)$ or $U_{3}(3)$. Let $P$ be a Sylow 2-subgroup of $H, Q$ a Sylow 3-subgroup of $H$ and $x$ an element of $G$ of order 7. If $K / H \simeq L_{2}(7)$, then $|Q|=3^{2}$. Since $(7,|A u t(Q)|)=1, x$ acts trivially on $Q$ and so 7 and 3 are joined, a contradiction. If $K / H \simeq L_{2}(8)$, then $P$ is of order $2^{3}$ and so $G$ has an element $x$ such that $\left|x^{G}\right| \leq 7$, a contradiction by [6]. Hence $K / H \simeq U_{3}(3)$. It is easy to see that $H=1$ since otherwise, $H$ is contained in $Z(G)$, a contradiction. Thus, $K \simeq U_{3}(3)$ and $G \simeq A u t\left(U_{3}(3)\right)$.

Lemma 3.2. Let $G$ be a group with $Z(G)=1$ and $M=\operatorname{Aut}\left(A_{6}\right)$. If $c s(G)=c s(M)$, then $G \simeq M$. 
Proof. We proceed the proof by several steps.

(1) By $[6], \operatorname{cs}(G)$ consists of $n_{1}=1, n_{2}=3^{2} \cdot 5, n_{3}=2^{4} \cdot 5, n_{4}=2 \cdot 3^{2} \cdot 5$, $n_{5}=2^{4} \cdot 3^{2}, n_{6}=2 \cdot 3 \cdot 5, n_{7}=2^{4} \cdot 3 \cdot 5, n_{8}=2^{2} \cdot 3^{2}, n_{9}=2^{2} \cdot 3^{2} \cdot 5$.

(2) By Lemma 2.1, we have that $\pi(G)=\pi(M)=\{2,3,5\}$.

(3) The Sylow 5-subgroups of $G$ are of order 5 .

Let $P$ be a Sylow 5-subgroup of $G$. Then, by Lemma 2.8, $P$ is elementary abelian since $5^{2}$ does not divide any element in $\operatorname{cs}(G)$. We assert that $P$ is of order 5. Assume that $5^{2}$ divide the order of $G$. Then, the centralizer of every element of $G$ contains an element of order 5. Let $y \in G$ such that $\left|y^{G}\right|=2^{4} \cdot 3 \cdot 5$ and $x$ an element of $C_{G}(y)$ of order 5. Since the Sylow 5subgroup of $G$ is elementary abelian, we have that 5 does not divide $\left|x^{G}\right|$ and consequently $x^{G}=2^{4} \cdot 3^{2}$ or $2^{2} \cdot 3^{2}$. If 5 does not divide $|y|$, then $C_{G}(x y)=C_{G}(x) \cap C_{G}(y)$, from which we can deduce that $2^{6} \cdot 3^{3} \cdot 5$ divide $(x y)^{G}$, which is impossible. If 5 divides $|y|$, then $y=y_{1} y_{2}$ with $\left|y_{1}\right|=5$ and $\left(\left|y_{1}\right|,\left|y_{2}\right|\right)=1$. It follows that $\left|y_{1}^{G}\right|$ should divide $\left|y^{G}\right|$, a contradiction. Hence the Sylow 5-subgroup of $G$ is of order 5 .

(4) $\mathrm{O}_{22^{\prime}}(G)=\mathrm{O}_{2}(G)$.

Write $K=O_{2}(G)$ and $\bar{G}=G / K$. Suppose that the statement is false. Then there is $r \in\{3,5\}$ such that $O_{r}(\bar{G}) \neq 1$. If $P=O_{5}(\bar{G}) \neq 1$, then $|P|=5$. Let $Q$ be a Sylow 3-subgroup of $G$ and $x$ an element of $Z(Q)$ of order 3. Then $\left|x^{G}\right|=n_{3}=2^{4} \cdot 5$ and so 5 does not divide $\left|C_{G}(x)\right|$ by Step 1 . Since $(3,5-1)=1$, we see that $\bar{x}$ acts trivially on $P$. Thus 5 divides $C_{\bar{G}}(\bar{x})$. Since $(3,|K|)=1$, we have that $C_{\bar{G}}(\bar{x})=C_{G}(x) K / K$ and so 5 divides $\left|C_{G}(x)\right|$, a contradiction. Now, we assume that $O_{3}(\bar{G}) \neq 1$. Put $V=\Omega_{1}\left(Z\left(O_{3}(\bar{G})\right)\right)$. Then $V$ is a nontrivial normal subgroup of $\bar{G}$. Let $y$ be an element of $G$ of order 5. Then $V=[V, \bar{y}] \times C_{V}(\bar{y})$. By Step $1,\left|V: C_{V}(\bar{y})\right|$ is at most $3^{2}$. It follows that $[[V, \bar{y}], \bar{y}]=1$, which implies that $[V, \bar{y}]=1$. Therefore $V=C_{V}(\bar{y})$. Let $Q$ be a Sylow 3 -subgroup of $\bar{G}$. Then $V \cap Z(Q) \neq 1$. Let $\bar{z}$ be an element of $V \cap Q$ of order 3. Then $\bar{y} \bar{z}=\bar{z} \bar{y}$. Since $(3,|K|)=1$, there exists a preimage $z$ of $\bar{z}$ in $G$ such that $z$ is contained in the center a Sylow $3-$ subgroup of $G$ and so 5 divides $\left|C_{G}(z)\right|$. But, by Step 1, this is impossible.

(5) $G \simeq \operatorname{Aut}\left(A_{6}\right)$.

Set $K=O_{2}(G)$ and $\bar{G}=G / K$. Then $\bar{G}$ is insoluble by Step 4 . Furthermore, we have that $M \leqslant \bar{G} \leqslant A u t(M)$, where $M=S_{1} \times S_{2} \times \cdots \times S_{k}$ is a direct product of non-abelian simples $S_{1}, S_{2}, \ldots, S_{k}$. Since $\pi(G)=\{2,3,5\}$ and the order of the Sylow 5 -subgroups of $G$ is 5 , we obtain that $k=1$, that is, $M$ is a non-abelian simple $K_{3}$-group. Hence $M$ is isomorphic to $A_{5}, A_{6}$ or $U_{4}(2)$.

If $M \simeq A_{5}$, then $G / K \simeq A_{5}$ or $A u t\left(A_{5}\right)$ and so $3^{2}$ does not divide $|G|$, a contradiction. 
Suppose that $M \simeq A_{6}$. Assume first that $\bar{G} \simeq A_{6}$ or $S_{6}$. Let $x$ be an element of $G$ of order 5 such that $n_{8}=\left|x^{G}\right|=2^{2} \cdot 3^{2}$. Then $\left|\bar{x}^{\bar{G}}\right|$ divides $2^{2} \cdot 3^{2}$. By [6], we have that $\left|\bar{x}^{\bar{G}}\right|=1$, which implies that $\bar{x} \leqslant Z(\bar{G})$. Therefore $\bar{x}=1$ for that $Z(\bar{G})$ is trivial and so $x \in K$, a contradiction.

Assume that $\bar{G} \simeq P G L_{2}(9)$. Pick $x \in G$ with $|x|=5$ such that $\left|x^{G}\right|=2^{2} \cdot 3^{2}$. Then, by [6], $\left|\bar{x}^{\bar{G}}\right|=1$ or $2^{2} \cdot 3^{2}$. As above, it is impossible that $\left|\bar{x}^{\bar{G}}\right|=1$. If $\left|\bar{x}^{\bar{G}}\right|=2^{2} \cdot 3^{2}$, then $x$ centralizes $K$. If $K \neq 1$, then $x$ centralizes an element of $G$ which lies in the center of a Sylow 2-subgroup of $G$, contrary to Step 1 . If $K=1$, then $G \simeq P G L_{2}(9)$, but $c s\left(P G L_{2}(9)\right) \neq c s(G)$ by [6], a contradiction.

Suppose that $\bar{G} \simeq M_{10}$. Let $x$ be an element of $G$ of order 5 such that $\left|x^{G}\right|=2^{4} \cdot 3^{2}$. Then $\left|\bar{x}^{\bar{G}}\right|=1$ or $2^{4} \cdot 3^{2}$ by [6]. Similar to the above case, we can derive a contradiction.

If $\bar{G} \simeq \operatorname{Aut}\left(A_{6}\right)$, then, similar to the forgoing argument, one can show that $K=1$ and so $G \simeq \operatorname{Aut}\left(A_{6}\right)$, as desired.

If $\bar{G} \simeq U_{4}(2)$, then $G$ has an element $x$ such that $\left|\bar{x}^{\bar{G}}\right|=2^{7} \cdot 3^{2} \cdot 5$. It follows that $\left|x^{G}\right|$ is divisible by $2^{7} \cdot 3^{2} \cdot 5$, which is impossible by Step 1 .

Thus, the proof is complete.

Lemma 3.3. Let $G$ be a group with $Z(G)=1$ and $M=A u t\left(U_{4}(2)\right)$. Suppose that $\operatorname{cs}(G)=c s(M)$ and $|G|_{p}=|M|_{p}$, where $|G|_{p}$ denotes the order of the Sylow $p$-subgroups of $G$ for $p \in\{2,3,5\}$. Then $G \simeq M$.

Proof. We proceed the proof by the following steps.

(1) $\operatorname{cs}(G)$ consists of $n_{1}=1, n_{2}=3^{2} \cdot 5, n_{3}=2 \cdot 3^{3} \cdot 5, n_{4}=2^{4} \cdot 5$, $n_{5}=2^{4} \cdot 3 \cdot 5, \quad n_{6}=2^{5} \cdot 3 \cdot 5, \quad n_{7}=2^{2} \cdot 3^{3} \cdot 5, \quad n_{8}=2^{3} \cdot 3^{4} \cdot 5, \quad n_{9}=2^{6} \cdot 3^{4}$, $n_{10}=2^{4} \cdot 3^{2} \cdot 5, \quad n_{11}=2^{5} \cdot 3^{2} \cdot 5, \quad n_{12}=2^{4} \cdot 3^{3} \cdot 5, \quad n_{13}=2^{7} \cdot 3^{2} \cdot 5, \quad n_{14}=$ $2^{5} \cdot 3^{3} \cdot 5, n_{15}=2^{2} \cdot 3^{2}, n_{16}=2^{2} \cdot 3^{4} \cdot 5, n_{17}=2^{4} \cdot 3^{4} \cdot 5$.

This follows from [6].

(2) $\pi(G)=\pi(M)=\{2,3,5\}$.

This follows from [6] and Lemma 2.1.

(3) $\mathrm{O}_{22^{\prime}}(G)=\mathrm{O}_{2}(G)$.

Write $K=O_{2}(G)$ and $\bar{G}=G / K$. Suppose that the assertion is false. Then $O_{r}(\bar{G}) \neq 1$, where $r \in\{3,5\}$. Assume first that $O_{5}(\bar{G}) \neq 1$. Then, $\left|O_{5}(\bar{G})\right|=5$. Let $x \in Z(Q)$ with $|x|>1$, where $Q$ is a Sylow 3-subgroup of $G$. Then $\left|x^{G}\right|=2^{4} \cdot 5$ by (1). Since $\bar{x}$ acts trivially on $O_{5}(\bar{G})$, we have that 5 divides the order of $C_{\bar{G}}(\bar{x})$, by which we conclude that 5 divides the order of $C_{G}(x)$. This is impossible by (1). Now assume that $O_{3}(\bar{G}) \neq 1$ and set $V=\Omega_{1}\left(Z\left(O_{3}(\bar{G})\right)\right)$. Let $y$ be an element of $G$ of order 5 . Then $\left|y^{G}\right|=2^{6} \cdot 3^{4}$ or $2^{2} \cdot 3^{2}$. Since $V=[V, \bar{y}] \times C_{V}(\bar{y})$, we have that $\left|V: C_{V}(\bar{y})\right| \leqslant 3^{4}$. If 
$\left|V: C_{V}(\bar{y})\right| \leqslant 3^{3}$, then $[[V, \bar{y}], \bar{y}]=1$ and so $[V, \bar{y}]=1$. Discussing as in Lemma 3.2, we see that this is impossible. If $\left|V: C_{V}(\bar{y})\right|=3^{4}$, then the Sylow 3-subgroups of $G$ is elementary abelian, which contradicts that $\left|x^{G}\right|=n_{13}=2^{7} \cdot 3^{2} \cdot 5$ for some 3-element $x \in G$.

(4) $G \simeq M$.

By (3), $\bar{G}$ is insoluble. Hence we have that $M \leqslant \bar{G} \leqslant A u t(M)$, where $M$ is a direct product of non-abelian simple groups. Since, by $(2) \pi(G)=$ $\pi(M)=\{2,3,5\}$, we know that $M$ is simple $K_{3}$-groups by (3). Thus, $M$ is isomorphic to $A_{5}, A_{6}$ or $U_{4}(2)$. If $M$ is isomorphic to $A_{5}$ or $A_{6}$, then $3^{3}$ does not divide the order of $G$, a contradiction by (1). Suppose that $M \simeq U_{4}(2)$. Then $\bar{G} \simeq U_{4}(2)$ or $U_{4}(2) .2$. If $\bar{G} \simeq U_{4}(2)$, then $Z(G)$ is nontrivial, a contradiction. If $\bar{G} \simeq U_{4}(2) .2$, then $G$ is isomorphic to $A u t\left(U_{4}(2)\right)$, as desired.

Proof of Theorem 1.2.

It follows from Lemmas 3.1-3.3.

Acknowledgments. The authors would like to express their sincere thanks for the referee's helpful comments.

\section{REFERENCES}

[1] N. Ahanjideh, On Thompson's conjecture for some finite simple groups, J. Algerba, 344 (2011), pp. 205-228.

[2] G. Y. Chen, On Thompson's Conjecture, PhD thesis, Sichuan University, Department of Mathematics, 1994.

[3] G. Y. Chen, On Thompson's cconjecture for sporadic simple groups, Proc. China Assoc. Sci. and Tech. First Academic Annual Meeting of Youths, Chinese Sci. and Tech. Press, Beijing 1992, pp. 1-6. (in Chinese)

[4] G. Y. Chen, On Thompson's conjecture, J. Algebra, 185 (1996), pp. 184-193.

[5] G. Y. Chen, Further reflections on Thompson's conjecture, J. Algebra, 218 (1999), pp. 276-285.

[6] J. H. Conway - R. T. Curtis - S. P. Norton - R. A. Parker - R. A. Wilson, Atlas of Finite Groups, Clarendon Press, Oxford, 1985.

[7] D. Gorenstein, Finite simple groups, Plenum Press, New York, 1982.

[8] W. B. Guo, The Theory of Classes of Groups, Science Press-Kluwer Academic Publishers, Beijing-New York-Dordrecht-Boston-London, 2000.

[9] A. Khosravi - B. Khosravi, A new characterization of almost sporadic groups, J. Algebra and Its Applications, 3 (2002), pp. 267-279.

[10] B. Khosravi - M. Khatami, A new characterization of $P G L(2, p)$ by its noncommuting graph, Bull. Malays. Math. Sci. Soc. (2), 34(3) (2011), pp. $665-674$. 
[11] V. D. Mazurov - E. I. Khukhro, Unsolved Problems in Group Theory: The Kourovka Notebook, 17th edition, Sobolev Institute of Mathematics, Novosibirsk, 2010.

[12] A. V. VAsIl'Ev, On Thompson's conjecture, Siberian Electronic Mathematical Reports, 6 (2009), pp. 457-464.

[13] J. S. Williams, Prime graph components of finite groups, J. Algebra, 69 (1981), pp. 487-513.

Manoscritto pervenuto in redazione il 2 Settembre 2013. 
